\title{
Unimanual muscle activation increases interhemispheric inhibition from the active to the resting hemisphere
}

\author{
Katrien Vercauteren, Tine Pleysier, Liesbet Van Belle, Stephan P. Swinnen, Nicole Wenderoth* \\ Motor Control Lab, Department of Biomedical Kinesiology, KU Leuven, Belgium
}

\section{A R T I C L E I N F O}

\section{Article history:}

Received 18 January 2008

Received in revised form 27 August 2008

Accepted 6 September 2008

\section{Keywords:}

Interhemispheric inhibition

Transcranial magnetic stimulation

Motor cortex

\begin{abstract}
A B S T R A C T
Interhemispheric inhibition ( $\mathrm{IHI}$ ) is an important mechanism to maximize the independent functioning of each hemisphere and is most likely mediated by transcallosal fibres. IHI can be investigated by paired pulse transcranial magnetic stimulation (TMS) whereby, in half of the trials, a test stimulus (TS) over one hemisphere is preceded by a conditioning stimulus (CS) over the other hemisphere. Whereas various studies have investigated $\mathrm{IHI}$ in rest, less is known about interhemispheric interactions during voluntary muscle activation. Here, we investigated the influence of tonic muscle activity ( $5 \%$ of the maximal voluntary contraction) in either the right wrist flexor or extensor versus rest on IHI from the active (left) to the resting (right) hemisphere. Our main finding was that tonic activation of the right wrist flexor, led to an increase in IHI from the active (dominant left) to the resting (non-dominant right) hemisphere as compared to rest. A control experiment employed the same design but CS intensity was lowered to match MEP amplitudes of the conditioning hand between active and rest conditions. This resulted in a relative decrease of IHI. It is hypothesized that functional regulation of IHI might prevent the occurrence of mirror activity in the primary motor cortex (M1) of the resting hemisphere and, thus, might play an important role in the execution of unimanual actions.
\end{abstract}

(c) 2008 Elsevier Ireland Ltd. All rights reserved.
The corpus callosum (CC) is the largest neural link that connects the cerebral hemispheres in mammals. It is a pathway for interhemispheric transfer of perceptual, sensory, motor, gnostic and other forms of information contributing to higher-order functions $[8,10,11,17,22]$. The CC promotes both interhemispheric facilitation and inhibition which play a major role in motor control and, particularly, in bimanual coordination [1,8-10,12,13,17].

Interhemispheric inhibition (IHI) can be studied by transcranial magnetic stimulation (TMS) which allows the non-invasive estimation of IHI in conscious human subjects [14] by applying paired pulse TMS as described by Ferbert et al. [9]. They showed that the amplitude of motor evoked potentials (MEP) produced by a test stimulus (TS) given over the motor cortex of one hemisphere was reduced when the TS is preceded by a conditioning stimulus (CS) over the homotopic area of the opposite hemisphere [9]. Several lines of research suggest that this protocol measures IHI mediated at cortical level and predominantly via transcallosal pathways $[5,6,11,28]$.

The effectiveness of double pulse TMS in probing IHI depends on several methodological parameters such as the interval between

\footnotetext{
* Corresponding author at: FABER, KU Leuven, Tervuursevest 101, 3001 Leuven (Heverlee), Belgium. Tel.: +32 16 329157; fax: +32 16329197.

E-mail address: Nicole.wenderoth@faber.kuleuven.be (N. Wenderoth).
}

both stimuli which is most efficient between 8 and $12 \mathrm{~ms}[3,9,13,15]$, but also the CS and TS intensity. The amount of IHI is positively correlated with suprathreshold CS intensity $[4,9,15]$ and negatively with the TS intensity (at $40-90 \%$ of stimulator output) [9]. Potential physiological factors affecting IHI are gender [4], direction of inhibition, i.e. from the dominant to the non-dominant hemisphere or versus the opposite direction $[4,7,9]$, and motor cortex excitability [7,29].

Previously, IHI was mainly investigated when subjects were at rest. However, in the context of movement control it is more interesting to know how $\mathrm{IHI}$ is modulated by voluntary muscular activity. With the present study, we aimed to extend the existing literature by investigating the influence of voluntary muscle activation of the right wrist extensor and flexor, respectively, on IHI from the left (active) to the right (resting) hemisphere. A recent study showed that IHI increases close to movement onset during a reaction time task [7].

Here we investigated, first, whether IHI is also increased during muscle activation and, second, whether IHI differs between homologous versus non-homologous muscles. Based on previous studies investigating IHI during passive wrist movements [29], we hypothesized that IHI increases only when the homologous but not the non-homologous muscle is active.

Thirteen healthy volunteers ( 7 female, mean age $22.86 \pm$ S.D. 2.39 years) participated in the main study. Six subjects ( 2 female, 


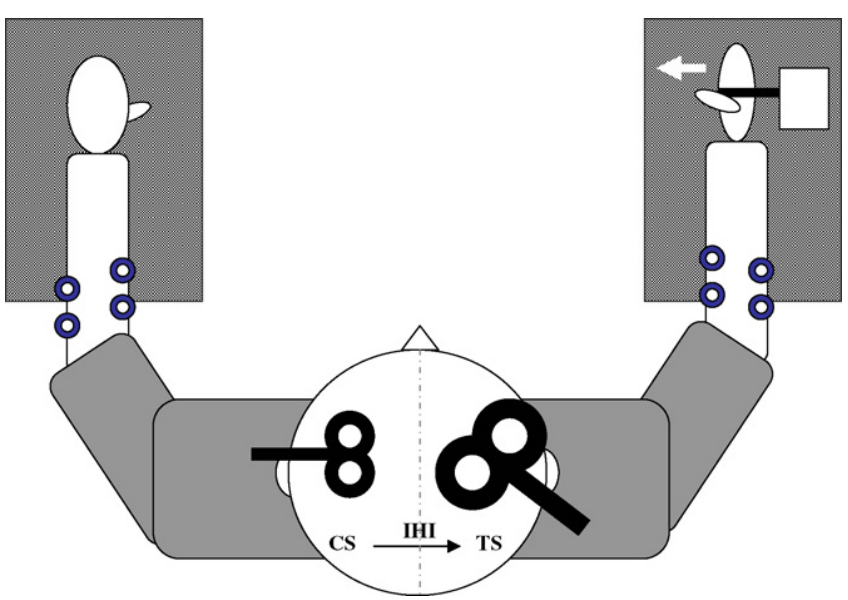

Fig. 1. General setup. The conditioning stimulus (CS) was delivered over the left and the test stimulus (TS) over the right hemisphere (ISI = $10 \mathrm{~ms}$ ) measuring interhemispheric inhibition (IHI) from the dominant left to the non-dominant right hemisphere. The subject held the left wrist always in a relaxed position, while the right wrist produced an isometric wrist flexion as measured by a load cell at the back of the hand or an isometric wrist extension measured with the load cell located in front of the hand palm (not shown).

mean age: $23.63 \pm$ S.D. 4.47 years) were included in a control experiment and one subject participated in both studies. All subjects were right-handed as assessed with the Edinburgh Handedness Inventory [21]. None had contra-indications to TMS [30] or a history of medical or psychiatric diseases. All subjects were informed about the purpose of this study and gave their written informed consent prior to participation which was approved by the local Ethics Committee according to the Declaration of Helsinki (1964).

Subjects were comfortably seated in a chair with their arms supported by two adjustable tables (Fig. 1). The left arm was kept relaxed and rested at the table with the wrist in pronation. The right forearm was fixed in a splint ensuring a neutral position between pronation and supination while the wrist could move freely. Two electrodes (Blue Sensor P-00-S, Ambu, Ølstykke, Denmark) were fixed to the muscle belly of the left and right extensor carpi radialis longus muscle (ECR) and the flexor carpi radialis muscle (FCR) to record an electromyogram (EMG) (Mespec 8000, Mega Electronics Ltd., Kuopio, Finland) at a frequency of $5 \mathrm{kHz}$. EMG data were band-pass filtered (30-1500 Hz) and saved for further analysis (CED Power 1401, Cambridge Electronic Design, Cambridge, UK en Signal 3.03). Subjects were instructed to fully relax and to avoid any obvious EMG activity of the non-moving muscles. EMG activity was displayed online on a computer screen in front of the subject was and continuously controlled by the experimenter.

Measurements were performed while either the ECR or FCR were isometrically activated at $5 \%$ maximum voluntary contraction (MVC) or at rest. Prior to the experiment, MVC was measured for the FCR $(36.20 \pm 10.50 \mathrm{~N})$ and ECR $(29.71 \pm 10.99 \mathrm{~N})$ by a force transducer (Tedea-Huntleigh, model 601). During the isometric conditions, the produced force was digitized at $5 \mathrm{kHz}$, and displayed as a cursor on a computer screen in front of the subject who had to align the cursor (representing the produced force) with a reference line indicating 5\% MVC of the activated muscle.

Subjects were instructed to completely relax the left body side, while performing either two active conditions, i.e. isometric 5\% MVC flexion (FLEX) or isometric 5\% MVC extension (EXT), or a rest condition (REST). Each trial lasted $5 \mathrm{~s}$ and, in the active conditions, a beep after $2.5 \mathrm{~s}$ indicated that subjects had to contract the investigated wrist muscle. At $4 \mathrm{~s}$, TMS was applied and subjects were instructed to relax immediately after the TMS pulse. In the REST condition, subjects relaxed the right hand during the whole trial.
Transcranial magnetic stimulation was delivered to M1 by 2 Magstim $200^{2}$ (Magstim Company Ltd., Carmarthenshire, UK) units. IHI from the dominant (left) to the non-dominant (right) hemisphere was measured by a double-pulse TMS protocol as described before [9]. The CS was delivered by a figure-of-eight coil (loop diameter: $50 \mathrm{~mm}$ ) which was positioned tangentially over M1 of the dominant (left) hemisphere such that the handle pointed sideward, i.e. $90^{\circ}$ away from the midline. The TS was delivered by another figure-of-eight coil (loop diameter: $70 \mathrm{~mm}$ ) placed tangentially over M1 of the non-dominant (right) hemisphere such that the handle pointed backwards and $45^{\circ}$ away from the midline. The hotspot for the FCR was located in each hemisphere and marked to ensure constant positioning throughout the experiment. Rest motor threshold (RMT) was determined for each hemisphere to the nearest $1 \%$ of the stimulator output that elicits a MEP of at least $50 \mu \mathrm{V}$ peak-to-peak amplitude in the relaxed FCR in at least 5 out of 10 consecutive stimuli. Subjects with a RMT above $60 \%$ stimulator output were excluded. Though we focused on stimulation parameters for the FCR, ECR parameters were assumed to be sufficiently similar due to the overlapping representations of forearm flexors and extensors such that MEPs could be evoked in both wrist muscles simultaneously [24,25]. During the main experiment, CS intensity was set at $110 \%$ of the FCR rest motor threshold in all conditions. For the FLEX, EXT, and REST condition the TS intensity was set at $110 \%$. However, previous research has shown that voluntary contractions can increase the corticospinal excitability of the non-involved, ipsilateral hemisphere [20]. In the context of our experiment, this represents a potential confound as the MEP size evoked by single pulse (non-conditioned) TMS would be smaller in REST than in the active conditions. Therefore, we introduced a second rest condition (RESTmatch) whereby the stimulation intensity was increased such that the non-conditioned MEP amplitude was matched to the active conditions to ensure that \%IHI was determined relative to comparable TS MEP amplitudes between conditions [16]. The mean intensity and standard deviation for the RESTmatch condition was $118.95 \pm 1.52 \%$ RMT. The ISI between the CS and TS was set to $10 \mathrm{~ms}$. For each experimental condition (FLEX, EXT, REST, and RESTmatch) subjects performed 5 series of 12 trials consisting of 6 single and 6 double pulse stimulations in randomized order. The inter-trial interval varied between 2 and $4 \mathrm{~s}$. The order of the conditions was randomized across subjects.

All EMG traces were visually inspected (Signal 3.03, Cambridge Electronic Design, Cambridge, UK) and trials were removed when (1) there was obvious background EMG, (2) the stimulation of the conditioning pulse resulted in a MEP (right FCR) below $50 \mu \mathrm{V}$, (3) the force level in the active conditions was higher than $7.5 \%$ or lower than $2.5 \%$ MVC. In total, $72 \%$ of all trials were correct and included in the further analysis. For each subject, the peak-to-peak amplitude of the MEP was determined for the FCR and the ECR of the test side (left arm) within an interval of 15-65 ms after the TMS stimulation. Individual MEP amplitudes were averaged for each muscle, condition, and single versus double pulse stimulation. The percentage IHI was determined by \%IHI $=[$ non-conditioned MEP amplitude - conditioned MEP amplitude $\times 100 /$ non-conditioned MEP amplitude] (thus high values represent a strong $\mathrm{IHI}$ and low values represent weak IHI). Background EMG was determined as the root-mean-square error (R.M.S.E.) value of the EMG signal in the last $50 \mathrm{~ms}$ interval before TMS stimulation. In the active conditions, the produced force was measured in a $1 \mathrm{~s}$ interval prior to TMS stimulation. One male subject was excluded from further analysis, because he exhibited facilitation in both muscles for almost every condition.

All statistical analyses were performed with Statistica 7.1 (StatSoft, Inc., Tulsa, USA). The R.M.S.E.-values of the background EMG of the left wrist muscles, MEP-amplitudes and percentage of 
inhibition were subjected to analyses of variance for repeated measurements (repeated measures ANOVA). Within-subject factors were muscle (ECR, FCR), TMS pulse (non-conditioned, conditioned) and condition (REST, RESTmatch, FLEX, and EXT). Gender was included as a between-subject factor. The criterion for statistical significance was $\alpha=0.05$. Significant effects were further tested with Fisher LSD post hoc tests.

In the control experiment general setup, EMG-recording, dataanalysis and statistics were the same as in the main experiment. Subjects had to isometrically flex the right wrist at 5\% MVC in the active condition (FLEX) or completely relax during REST. CS intensity was set to $140 \%$ of the FCR RMT in REST and FLEX condition. Additionally, a second FLEX condition (FLEXmatch) was tested whereby the stimulation intensity of CS was adjusted such that the MEP amplitude in the active FCR was comparable between FLEXmatch and REST. Mean CS intensity for the FLEXmatch condition was $94.82 \pm 21.23 \%$ RMT. During the REST condition, the TS intensity was set to $120 \%$ RMT and was adjusted for both FLEX conditions such that the non-conditioned MEP amplitude of the resting FCR was matched to REST. For the FLEX and FLEXmatch condition, TS intensity was respectively $111.57 \pm 9.58 \%$ RMT and $111.83 \pm 9.96 \%$ RMT. For each condition (FLEX, FLEXmatch and REST) subjects performed 3 series of 12 trials whereby 6 single and 6 double pulse stimulations were given in a randomized order. In this experiment, we used higher CS intensities than in the main experiment, because MEPs of the right FCR had to be sufficiently large at REST to allow a successful matching with the active condition. We additionally increased TS intensity such that the level of IHI in REST was similar in both experiments.

Subjects complied well with the demands of the task and the forces determined for FLEX and EXT were $5.53 \pm 0.21 \%$ MVC and $5.45 \pm 0.29 \% \mathrm{MVC}$, respectively. There was no significant difference in force between both active conditions $\left(F_{(1,9)}=1.93 ; p=0.20\right)$.

Fig. 2A depicts the mean MEP amplitudes of the left wrist muscles as evoked by single pulse TMS to the right hemisphere. MEP amplitudes were generally larger in the ECR than in the FCR $\left(F_{(1,10)}=5.643 ; p=0.039\right)$. As expected, the MEP amplitude at REST was significantly lower (condition main effect $F_{(3,30)}=8.386$; $p<0.001)$ than in FLEX $(p=0.001)$, EXT $(p=0.003)$, and RESTmatch $(p=0.002)$. Importantly, no significant differences were found between the MEP amplitude in the RESTmatch condition and the active conditions FLEX and EXT ( $p>0.77)$.

Mean \%IHI was significantly higher in the active than in the rest conditions (condition main effect: $\left(F_{(3,30)}=4.8, p<0.01\right)$ (Fig. $2 \mathrm{~B}$ ). Additionally, \% IHI was generally larger and more modulated by the experimental conditions for the FCR than the ECR as indicated by a significant main effect of Muscle $\left(F_{(1,10)}=6.69, p<0.05\right)$ as well as a significant Muscle $\times$ Condition interaction $\left(F_{(3,30)}=3.17, p<0.05\right)$. Interestingly, \%IHI in the FCR was substantially lower during REST and RESTmatch than during FLEX and EXT. This was confirmed by post hoc tests $(p<0.05)$ for all comparisons. \%IHI in the ECR was lower during RESTmatch than during FLEX $(p<0.05)$. There was also a tendency for \%IHI to be smaller in EXT than in FLEX $(p=0.058)$.

\%IHI was generally larger in males than in females (gender main effect $\left.F_{(1,10)}=8.876, p=0.014\right)$ and also that the difference in \%IHI for FCR versus ECR, was much larger in males (\%IHI FCR: $45.3 \pm 10.2 \%$, \%IHI ECR $28.9 \pm 3.2 \%$ ) than in females (\%IHI FCR $12.6 \pm 10.6 \%$, \% IHI ECR $11.8 \pm 4.13 \%$ ) (Muscle $\times$ Gender interaction $\left.F_{(1,10)}=5.53, p<0.05\right)$.

Background EMG of the left hand was very small (below $2.5 \mu \mathrm{V}$ RMS), indicating that subjects were relaxed. However, statistics revealed a small but significant difference in background EMG in the REST (mean: $1.70 \pm$ S.D. $0.32 \mu \mathrm{V}$ ) as compared to the EXT (mean: $1.9 \pm$ S.D. $0.39 \mu \mathrm{V}$ ) and RESTmatch condition (mean: $1.89 \pm$ S.D. $0.43 \mu \mathrm{V})\left(F_{(3,33)}=4.510 ; p<0.01\right)$. Unexpectedly, EMG values were larger in the single pulse (mean: $1.94 \pm$ S.D. $0.58 \mu \mathrm{V}$ ) than in the double pulse condition (mean: $1.76 \pm$ S.D. $0.49 \mu \mathrm{V})\left(F_{(1,11)}=12.002\right.$; $p<0.01$ ).

Non-conditioned MEP amplitudes evoked by the TS in the resting (left) FCR were matched between FLEX $(0.40 \pm 0.19 \mathrm{mV})$, FLEXmatch $(0.43 \pm 0.18 \mathrm{mV})$ and REST $(0.44 \pm 0.19 \mathrm{mV})$ (condition main effect: $\left.\left(F_{(2,10)}=1.11, p>0.3\right)\right)$ in the control experiment. Also MEP amplitudes evoked by the CS in the active (right) hand were matched between the FLEXmatch $(0.51 \pm 0.27 \mathrm{mV})$ and REST condition $(0.53 \pm 0.14 \mathrm{mV})$ whereas MEP amplitudes were significantly larger for the unmatched FLEX condition $\left(F_{(2,10)}=27.27, p<0.01\right)$.

Matching the MEP amplitude evoked by the CS had a significant effect on \% IHI. As shown in Fig. 2C, IHI increased relative to rest in the FLEX condition but decreased relative to rest in the FLEXmatch condition. Accordingly, statistics revealed a significant Condition main effect $\left(F_{(2,10)}=11.14, p<0.01\right)$ and post hoc tests indicated that \%IHI was significantly lower in FLEXmatch than in FLEX and REST $(p<0.01)$. Background EMG was comparable between conditions $\left(F_{(2,10)}=0.95, p>0.4\right)$.

We investigated the influence of tonic muscle activity versus rest on IHI from the dominant left to the non-dominant right hemisphere. Our main finding was that tonic activation of the right FCR or ECR led to an increase in IHI from the active (dominant left) to the resting (non-dominant right) hemisphere as compared to the rest condition. Effects were smaller for the ECR, probably because all stimulation parameters were prioritized for the FCR. Our results extend recent work showing an increase of IHI from the active to the resting hemisphere before movement onset in a RT task [7]. We also included an additional rest control condition (REST-
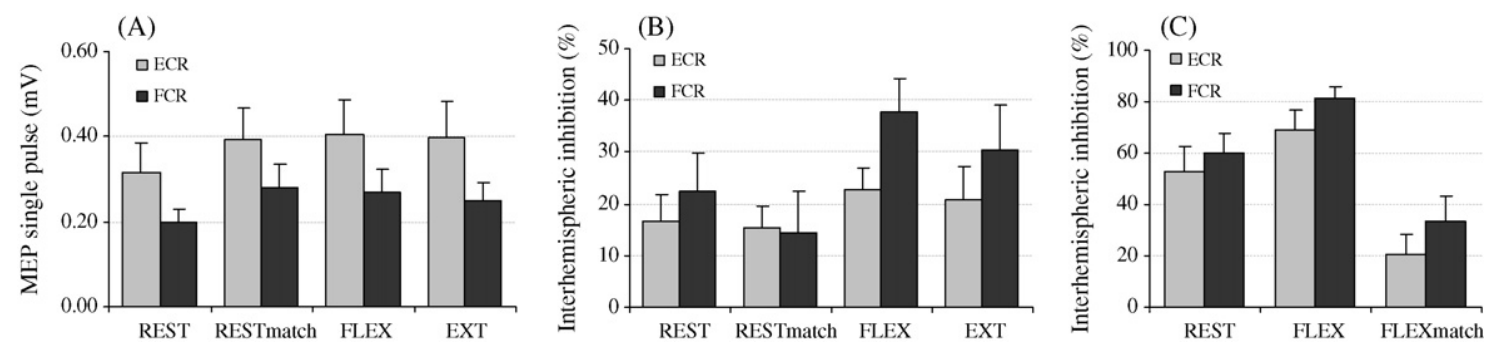

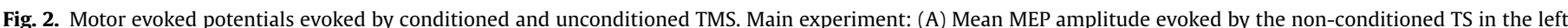

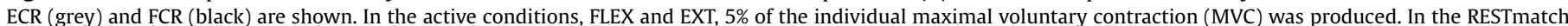

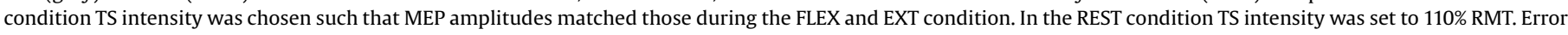

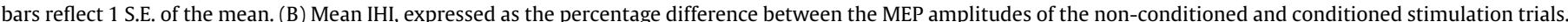

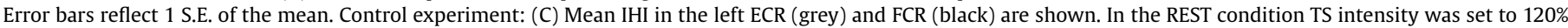

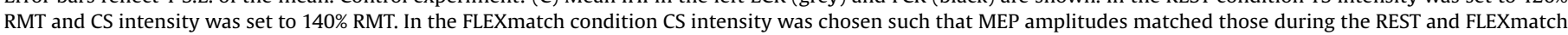
condition. Error bars reflect 1 S.E. of the mean. 
match) which showed that our findings were not confounded by changes in the corticospinal excitability of the right (resting) hemisphere as a consequence of muscle activation in the right hand (a phenomenon known as ipsilateral facilitation). Additionally, we observed a tendency that IHI increased even more between homologous as compared to non-homologous muscles. Similar results were obtained by Warbrooke and Byblow [29] who moved subjects' right wrist passively while stimulating the left FCR. They found that IHI was stronger during the flexion than during the extension subcycle of the passively moved hand, supporting the notion that IHI is different between homologous versus non-homologous muscles [29].

Our results are in general agreement with a recent model describing interactions between interhemispheric and intracortical inhibitory circuits $[2,18,19]$, such that a decrease in intracortical inhibition (ICI) leads to an increase of IHI, and vice versa. Applying this model to our experiment, it is tempting to speculate that the isometric activation of the right hand's wrist muscles led to a general release of ICI in the left (active) hemisphere. This, in turn, induced an increase of IHI to the motor area of the right (resting) hemisphere as shown by our results (Fig. 2B). This is of functional relevance as IHI might play an important role in suppressing unwanted mirror movements during intended unimanual movements by inhibiting the contralateral M1. In addition, the model predicts that an increase in IHI might affect inhibitory circuits of the right (resting) hemisphere, such that ICI is released which, most likely, contributed to the slight increase of corticospinal excitability, as reflected by the increased amplitude of the non-conditioned MEPs during the active conditions (Fig. 2A) $[23,26]$.

Even though the above interpretation is congruent with previous results and current models on interhemispheric inhibition, some caution is required. In the control experiment we matched the MEP amplitude evoked by the CS in the right hand between the REST and FLEXmatch condition, adjusting stimulation parameters for measuring IHI to the higher corticospinal output resulting from force production. In this condition, \%IHI was lower during isometric activation than during the rest and non-matched FLEX condition. Analogous results were obtained in a recent study by Perez and Cohen [23], whereby force levels from 10\% to 70\% MVC were used to investigate the interaction between interhemispheric and intracortical inhibition.

There are different interpretations of the differential influence of motor activity on IHI when tested with and without adjustment of CS intensity. At the one hand, one might argue that "true" IHI was reduced by the isometric contraction (as indicated by its reduction for adapted CS intensities). However, this is unlikely for several reasons. First, IHI is positively correlated with suprathreshold CS intensity, i.e., IHI is generally smaller for lower CS intensities $[4,9,15]$. Second, tonic contractions modulate IHI differentially in young than in elderly subjects, indicating that voluntary contraction has a modulating influence on IHI beyond methodological confounds [27]. Finally, experiments at rest have revealed similar modulations of IHI close to movement onset during a reaction time task [7]. At the other hand, the differences between the main and control experiment might reveal new insights into the interplay of excitatory and inhibitory neural circuits. Voluntary contractions activate muscle specific excitatory neurons, mediating an increase of corticospinal excitability. In the control experiment, we compensated for this facilitating effect by reducing CS intensity from $140 \%$ to $94.83 \% \pm 21.23$ RMT and, consequently, the measured level of IHI decreased. Together, both experiments suggest that the effect of measured IHI from the active (dominant left) to the resting (non-dominant right) hemisphere was mainly mediated by the active hemisphere and did not result from changes in the resting hemisphere. Moreover, it confirms that the activity of transcallosal neurons mediating IHI is controlled similarly to corticospinal neurons. Surprisingly, with adjustment of the CS intensity, \%IHI was significantly smaller than in REST as if an "over-correction" in relation to the corticospinal output had occurred. This might suggest that the relation between IHI and the activity of excitatory neurons might be non-linear at low force levels.

\section{Acknowledgements}

This paper was supported by a research grant G.0577.06 from the Fund for Scientific Research-Flanders (FWO-Flanders) and the KU Leuven Research Council (CREA).

\section{References}

[1] J.S. Bloom, G.W. Hynd, The role of the corpus callosum in interhemispheric transfer of information: excitation or inhibition? Neuropsychol. Rev. 15 (2005) 59-71.

[2] R. Chen, Interactions between inhibitory and excitatory circuits in the human motor cortex, Exp. Brain Res. 154 (2004) 1-10.

[3] R. Chen, D. Yung, J.Y. Li, Organization of ipsilateral excitatory and inhibitory pathways in the human motor cortex, J. Neurophysiol. 89 (2003) $1256-1264$.

[4] L. De Gennaro, M. Bertini, F. Pauri, R. Cristiani, G. Curcio, M. Ferrara, P.M. Rossini, Callosal effects of transcranial magnetic stimulation (TMS): the influence of gender and stimulus parameters, Neurosci. Res. 48 (2004) 129-137.

[5] L. De Gennaro, R. Cristiani, M. Bertini, G. Curcio, M. Ferrara, F. Fratello, V. Romei, P.M. Rossini, Handedness is mainly associated with an asymmetry of corticospinal excitability and not of transcallosal inhibition, Clin. Neurophysiol. 115 (2004) 1305-1312.

[6] V. Di Lazzaro, A. Oliviero, P. Profice, A. Insola, P. Mazzone, P. Tonali, J.C. Rothwell Direct demonstration of interhemispheric inhibition of the human motor cortex produced by transcranial magnetic stimulation, Exp. Brain Res. 124 (1999) 520-524.

[7] J. Duque, N. Murase, P. Celnik, F. Hummel, M. Harris-Love, R. Mazzocchio, E. Olivier, L.G. Cohen, Intermanual differences in movement-related interhemispheric inhibition, J. Cogn. Neurosci. 19 (2007) 204-213.

[8] J.C. Eliassen, K. Baynes, M.S. Gazzaniga, Anterior and posterior callosal contributions to simultaneous bimanual movements of the hands and fingers, Brain 123 (Pt 12) (2000) 2501-2511.

[9] A. Ferbert, A. Priori, J.C. Rothwell, B.L. Day, J.G. Colebatch, C.D. Marsden, Interhemispheric inhibition of the human motor cortex, J. Physiol. 453 (1992) 525-546.

[10] E.A. Franz, K.E. Waldie, M.J. Smith, The effect of callosotomy on novel versus familiar bimanual actions: a neural dissociation between controlled and automatic processes? Psychol. Sci. 11 (2000) 82-85

[11] M.S. Gazzaniga, Forty-five years of split-brain research and still going strong, Nat. Rev. Neurosci. 6 (2005) 653-659.

[12] M.S. Gazzaniga, Cerebral specialization and interhemispheric communication: does the corpus callosum enable the human condition? Brain 123 (Pt 7)(2000) $1293-1326$.

[13] C. Gerloff, L.G. Cohen, M.K. Floeter, R. Chen, B. Corwell, M. Hallett, Inhibitory influence of the ipsilateral motor cortex on responses to stimulation of the human cortex and pyramidal tract, J. Physiol. 510 (Pt 1) (1998) 249-259.

[14] M. Hallett, Transcranial magnetic stimulation and the human brain, Nature 406 (2000) 147-150.

[15] R. Hanajima, Y. Ugawa, K. Machii, H. Mochizuki, Y. Terao, H. Enomoto, T Furubayashi, Y. Shiio, H. Uesugi, I. Kanazawa, Interhemispheric facilitation of the hand motor area in humans, J. Physiol. 531 (2001) 849-859.

[16] M.L. Harris-Love, M.A. Perez, R. Chen, L.G. Cohen, Interhemispheric inhibition in distal and proximal arm representations in the primary motor cortex, $\mathrm{J}$. Neurophysiol. 97 (2007) 2511-2515.

[17] S.W. Kennerley, J. Diedrichsen, E. Hazeltine, A. Semjen, R.B. Ivry, Callosotomy patients exhibit temporal uncoupling during continuous bimanual movements Nat. Neurosci. 5 (2002) 376-381.

[18] S. Kukaswadia, A. Wagle-Shukla, F. Morgante, C. Gunraj, R. Chen, Interactions between long latency afferent inhibition and interhemispheric inhibitions in the human motor cortex, J. Physiol. 563 (2005) 915-924.

[19] H. Lee, C. Gunraj, R. Chen, The effects of inhibitory and facilitatory intracortical circuits on interhemispheric inhibition in the human motor cortex, J. Physiol. 580 (2007) 1021-1032.

[20] W. Muellbacher, S. Facchini, B. Boroojerdi, M. Hallett, Changes in motor cortex excitability during ipsilateral hand muscle activation in humans, Clin. Neurophysiol. 111 (2000) 344-349.

[21] R.C. Oldfield, The assessment and analysis of handedness: the Edinburgh inventory, Neuropsychologia 9 (1971) 97-113.

[22] L.K. Paul, W.S. Brown, R. Adolphs, J.M. Tyszka, L.J. Richards, P. Mukherjee, E.H Sherr, Agenesis of the corpus callosum: genetic, developmental and functional aspects of connectivity, Nat. Rev. Neurosci. 8 (2007) 287-299. 
[23] M.A. Perez, L.G. Cohen, Mechanisms underlying functional changes in the primary motor cortex ipsilateral to an active hand, J. Neurosci. 28 (2008) 5631-5640.

[24] M.H. Schieber, How might the motor cortex individuate movements? Trends Neurosci. 13 (1990) 440-445.

[25] M.H. Schieber, Constraints on somatotopic organization in the primary motor cortex, J. Neurophysiol. 86 (2001) 2125-2143.

[26] P. Talelli, A. Ewas, W. Waddingham, J.C. Rothwell, N.S. Ward, Neural corre lates of age-related changes in cortical neurophysiology, Neuroimage 40 (2008) 1772-1781.

[27] P. Talelli, W. Waddingham, A. Ewas, J.C. Rothwell, N.S. Ward, The effect of age on task-related modulation of interhemispheric balance, Exp. Brain Res. 186 (2008) 59-66.
[28] M. Wahl, B. Lauterbach-Soon, E. Hattingen, P. Jung, O. Singer, S. Volz, J.C. Klein, H. Steinmetz, U. Ziemann, Human motor corpus callosum: topography, somatotopy, and link between microstructure and function, J. Neurosci. 27 (2007) $12132-12138$.

[29] S.A. Warbrooke, W.D. Byblow, Modulation of interhemispheric inhibition during passive movement of the upper limb reflects changes in motor cortical excitability, Exp. Brain Res. 156 (2004) 11-19.

[30] E.M. Wassermann, Risk and safety of repetitive transcranial magnetic stimulation: report and suggested guidelines from the International Workshop on the Safety of Repetitive Transcranial Magnetic Stimulation, June 5-7, 1996, Electroencephalogr. Clin. Neurophysiol. 108 (1998) $1-16$. 